\title{
Isotropic Brillouin Spectra of Liquids Having an Internal Degree of Freedom.
}

\author{
A. Patkowski and J. Gapinski \\ Institute of Physics, A. Mickiewicz University, Umultowska 85, 61-614, Poznan, Poland, \\ G. Meier and H. Kriegs \\ Institut für Festkörperforschung, Forschungszentrum Jülich, 52425 Jülich, Germany
}

\begin{abstract}
Isotropic Brillouin spectra of the two chemically similar van der Waals glass forming liquids: 1,1'-di(4-methoxy-5-methylphenyl)cyclohexane (BMMPC) and 1,1'-bis(p-methoxyphenyl)cyclohexane (BMPC) and ortho-terphenyl (OTP) were studied in a broad temperature and pressure range in order to characterize the effect of intra-molecular relaxations on damping of longitudinal phonons. Such relaxations are present in BMPC, while in BMMPC and OTP they are strongly hindered. We show that in BMPC (with strong internal relaxations) the damping (broadening) of longitudinal phonons (Brillouin peaks) is much stronger than in BMMPC and OTP (with weaker internal relaxations). The contributions of the intramolecular and structural relaxations to the phonon damping can be separated using high pressure, due to their very different pressure dependence. We show that internal relaxations strongly contribute to the damping of longitudinal phonons at all temperatures and should be taken into account in theoretical models describing the Brillouin spectra of supercooled liquids.
\end{abstract}




\section{Introduction}

Scattering of laser light on propagating density fluctuations (longitudinal and transverse phonons) in a liquid is studied by means of the Rayleigh-Brillouin spectroscopy. If both incident and scattered light are polarized perpendicular to the scattering plane (VV geometry) the Rayleigh-Brillouin spectrum consists of the central Rayleigh line and two Brillouin lines symmetrically shifted by $\pm \omega_{B}$ of the width of $\Gamma_{B}$. The shift $\omega_{B}$ is related to the sound velocity $u$ via $\omega_{B}=q u$, where the scattering vector $q=|\boldsymbol{q}|=(4 \pi n \sin \theta / 2) / \lambda, \lambda$ is the wavelength of the incident light in vacuum, $n$ is the refractive index of the medium and $\theta$ is the scattering angle. The width of the Brillouin line $\Gamma_{B}$ depends on the damping of phonons and can be related to the relaxation processes in the liquid. Obviously, the closer the relaxation time to the inverse phonon frequency $1 / \omega_{\mathbf{B}}$ the more efficient is the damping. According to the predictions of the classical acoustic relaxation theory for a single relaxation process, the maximum of the sound attenuation coefficient $2 \pi \Gamma_{B} / \omega_{B}$ and of the damping occurs when $\omega_{B} \tau_{s}=1$. The structural relaxation time $\tau_{s}$ in supercooled liquids strongly depends on temperature $(T)$ and pressure $(P)$. Thus, the damping of phonons due to structural relaxations will depend strongly on $T$ and $P$. On the other hand, the temperature and pressure dependencies of internal relaxation times are much weaker. The relaxation times of internal processes are, at all temperatures, much closer to $1 / \omega_{\mathbf{B}}$ than that of structural relaxations. Thus, one would expect a substantial contribution of internal relaxations to the damping of phonons. This contribution will depend on temperature mainly because of the change of the amplitude of internal processes, since their relaxation times do not change much.

In the case of supercooled liquids the situation is much more complicated due to the presence of several relaxation processes. Additional complications might result from the fact that intramolecular relaxation processes in the liquid might also contribute to the damping of phonons. 
Theoretical models describing the polarized (VV) and depolarized (VH) Brillouin spectra of light scattered from supercooled liquids in terms of structural relaxation processes have been developed and tested experimentally ${ }^{1,2}$. In molecular liquids in addition to the intermolecular (structural) relaxations also intramolecular relaxations (overdamped vibrations) might be present. These intramolecular relaxations in supercooled liquids are usually not well characterized and their effect on the damping of phonons is much less studied and understood. A theory was developed ${ }^{3}$ for molecular liquids with intramolecular relaxations in the limit of weak coupling of these relaxations to the translational molecular motions. It was shown, that the polarized (VV) Brillouin lines, which are due to longitudinal phonons, are additionally broadened, while the depolarized $(\mathrm{VH})$ Brillouin lines, due to transverse phonons, are unaffected by internal relaxations. In order to test this theory experimentally, one needs materials with and without well characterized intramolecular relaxations. Additionally, one needs to find ways to separate the contributions of the intramolecular and structural relaxations to the damping of phonons and broadening of the corresponding Brillouin lines.

Such a separation can be achieved on the basis of different pressure dependence of intra- and inter-molecular (structural) relaxations. It is well established, that the structural relaxation in supercooled liquids depends strongly on pressure. A typical shift of the glass transition temperature with pressure $d T_{g} / d P$ amounts to $20-30 \mathrm{~K} / \mathrm{kbar}$. On the other hand, there is only few experimental data on the pressure dependence of internal relaxations in liquids ${ }^{4-7}$. However, due to their intra-molecular character, it is reasonable to expect that their pressure dependence would be much weaker and probably negligible in the pressure range of $1-2000$ bar. In polymers with side chain or side group relaxations ( $\beta$-relaxation) the value for $\mathrm{X}_{T}=\frac{\partial \log \tau_{\beta}}{\partial P}$ is usually about $2-5 \times 10^{-4} \operatorname{bar}^{-1}$ and thus two orders of magnitude smaller than the respective value for the $\alpha$-relaxation. ${ }^{4-6}$ In a study on non-polymeric glass formers ${ }^{7}$ this coefficient was found to be even smaller in agreement with the expectation that in the absence 
of chain effects the intra-molecular character of the $\beta$-relaxation is even more pronounced. Thus, the contributions of the structural and internal relaxations to the broadening (damping) of Brilloiun lines (phonons) can be estimated at any temperature from the pressure dependence of $\Gamma_{\mathbf{B}}(\mathrm{P})$. If the above considerations are correct, then at temperatures above $T_{\boldsymbol{g}}$, $\Gamma_{\boldsymbol{B}}(P)$ will first decrease with increasing pressure, due to substantial slowing down of the structural relaxation, until $T_{g}\left(P_{g}\right)$ is reached, and then level off at the high pressure plateau. This plateau corresponds to the contribution of the internal relaxations to the $\Gamma_{B}$. Consequently, in the supercooled liquid range the remaining width above the high pressure plateau is due to the contribution of the structural relaxations to the Brillouin line width and the damping of phonons.

To the best of our knowledge, no systematic study of the effect of intramolecular relaxations in supercooled molecular liquids on the damping of phonons has been performed. Extensive studies of relaxation processes and the Brillouin spectra were performed for ortho-terphenyl (OTP) in a broad temperature range ${ }^{8-12}$. Besides the $\alpha$-relaxation a fast process of practically temperature independent relaxation time in the picosecond range was found ${ }^{8-11}$. It was present both in liquid and crystalline samples and was assigned to intramolecular relaxation because, according to the predictions of the theory ${ }^{3}$, it was contributing to the damping of longitudinal but not transverse phonons. A similar process was also found in molecular dynamics simulation studies of $\mathrm{OTP}^{13}$. In temperature dependent Brillouin studies it was shown ${ }^{14}$ that while at the liquid-glass transition temperature $T_{g}$ the width of transverse Brillouin lines approaches zero, the width of the longitudinal Brillouin lines remains small, but still different from zero, due to the contribution of the internal relaxations. Generally, however, it was not possible to separate the contributions of the structural and internal relaxations to the damping (broadening) of phonons (Brillouin lines) at temperatures higher than $T_{\boldsymbol{g}}$. 
Structural (inter-) and intra-molecular relaxations were identified on a molecular level and studied in two chemically very similar (Fig.1) van der Waals supercooled liquids 1,1'-di(4-methoxy-5-methylphenyl)cyclohexane (BMMPC) and 1,1'-bis(p-methoxyphenyl)cyclohexane (BMPC). ${ }^{15,16}$ These liquids have a very similar chemical structure, the only difference being the presence of an additional methyl group in the ortho position in both phenyl rings of BMMPC. In BMPC in addition to the structural relaxation two intramolecular processes are present: the phenyl flip and the rotation of the $-\mathrm{OCH}_{3}$ group. In $\mathrm{BMMPC}$ the internal processes are strongly suppressed due to the chemical modification. This well identified difference in the internal dynamics makes this pair of molecular liquids a perfect choice to study the effects of internal relaxations on damping of phonons.

Recently ${ }^{17}$ we have shown, that in the case of molecules with weak internal relaxations (BMMPC) only the damping of the longitudinal phonons is affected, in agreement with the theoretical predictions for the weak coupling limit ${ }^{3}$. Strong internal relaxations (BMPC) contribute to damping of both longitudinal and transverse phonons. No theoretical model is available to explain this effect. We also demonstrated that the contribution of structural and internal relaxations to the broadening of the Brillouin lines can be separated by studying the pressure dependence of the Brillouin spectra.

In this work we present systematic studies of propagation and damping of longitudinal phonons in BMPC and BMMPC in a broad temperature and pressure range. The results obtained for these two liquids are compared with corresponding literature and measured data for a well characterized molecular supercooled liquid OTP. We show that the contribution of internal relaxations to the broadening of the Brillouin spectra can be estimated at any temperature from the pressure dependent studies. By comparing the data obtained for a liquid with well identified strong internal relaxations (BMPC) with those for a liquid with weak 
internal processes we show the role of intramolecular processes in the damping of longitudinal phonons.

\section{Theoretical background}

The isotropic Brillouin spectrum can be calculated from the experimental polarized (VV) and depolarized (VH) Brillouin spectra:

$$
I_{\text {Iso }}(q, \omega)=I_{V V}(q, \omega)-\frac{1}{d} I_{V H}(q, \omega)
$$

where $\mathrm{d}$ is the depolarization ratio. The isotropic spectrum decays to zero at frequencies beyond the Brillouin peak $(\omega / 2 \pi>40 \mathrm{GHz})$. Thus, the depolarization ratio can be obtained from these high frequency parts of the VV and VH Brillouin spectra.

The experimental isotropic Brillouin spectrum can be expressed as a convolution of the dynamic structure factor $\mathrm{S}(\mathrm{q}, \omega)$ (describing the spectrum of density fluctuations in the system) and the instrumental resolution function $R(\omega)$ :

$$
I_{\text {Iso }}(q, \omega)=I_{0} \int R\left(\omega^{\prime}\right) S\left(q, \omega-\omega^{\prime}\right) d \omega^{\prime}
$$

where $\mathrm{I}_{0}$ is a constant. The dynamic structure factor can be expressed in terms of the memory function $\mathrm{m}(\mathrm{q}, \omega)$ using simple generalized hydrodynamic formalism ${ }^{11,18}$

$$
S(q, \omega)=\frac{2 v_{0}^{2} q^{2}}{\omega} \operatorname{Im}\left[\omega^{2}-\omega_{T}^{2}(q)-i \omega m_{q}(\omega)\right]^{-1}
$$

where $\mathrm{v}_{0}=\mathrm{k}_{\mathrm{B}} \mathrm{T} / \mathrm{M},\left(\mathrm{M}\right.$ is the molecular mass of the scattering molecule), $\omega_{\mathrm{T}}{ }_{(\mathrm{q} \rightarrow 0)}=\mathrm{q}^{2} \mathrm{c}_{0}{ }^{2} / \gamma, \mathrm{c}_{0}$ is the adiabatic sound velocity and $\gamma=\mathrm{c}_{\mathrm{P}} / \mathrm{c}_{\mathrm{V}}$ is the ratio of specific heats at constant pressure and volume, respectively. 
In the case of OTP the memory function $K(\omega)$, related to $m(\omega)$ in eq. 3 simply by neglecting there the contribution of the thermal diffusivity term, contains terms related to three relaxation processes $^{11}$ : (i) the structural relaxation contribution in the form of the Cole-Davidson process, (ii) fast relaxation in the form of the Debye process, and (iii) a constant in frequency microscopic or instantaneous process:

$$
K(\omega)=\frac{q^{2} \Delta_{\alpha}^{2}}{i \omega \rho}\left[1-\frac{1}{\left(1+i \omega \tau_{\alpha}\right)^{\beta_{C D}}}\right]+\frac{q^{2} \Delta_{f}^{2} \tau_{f}}{\rho\left(1+i \omega \tau_{f}\right)}+\gamma_{0} q^{2}
$$

where $\rho$ is the liquid density, $\Delta_{\alpha}, \Delta_{\mathrm{f}}$ and $\tau_{\alpha}, \tau_{\mathrm{f}}$ are the amplitudes and relaxation times of the structural $(\alpha)$ and fast (f) relaxations, respectively, $\beta_{\mathrm{CD}}$ is the Cole-Davidson exponent and $\gamma_{0}$ is the amplitude of the microscopic process. Using this phenomenological approach it was possible to fit the shape of the isotropic Brillouin spectra of some glass forming liquids. ${ }^{11}$ In this kind of analysis all relaxation processes are added up in the memory function regardless of their physical origin. Thus, no information is obtained on their physical nature.

A theory describing explicitly the contribution of internal relaxations to the damping (broadening) of phonons (Brillouin lines) was developed by Zwanzig ${ }^{3}$ in the limit of weak coupling of internal processes to the centre-of-mass motions. In this limit it is assumed that the contribution due to coupling of internal and centre-of mass motions to the total Hamiltonian (consisting of parts related to the centre-of-mass and internal motions) is very small $^{3}$. The consequences of this limit are that, to a good approximation, the averages and time derivatives of functions depending on translational and internal variables can be calculated independently.

The longitudinal phonon linewidth is given in the classical hydrodynamic theory by ${ }^{19}$ :

$$
\Gamma_{B}=\frac{q^{2}}{2 M \rho}\left(\eta_{v}+\frac{4}{3} \eta\right)
$$


after neglecting the contribution due to the thermal conductivity term. Here $\rho$ is the number density of molecules in the liquid and $\eta$ and $\eta_{v}$ are the shear and bulk viscosities, respectively. In the weak coupling limit, the bulk viscosity, determining the width of the Brillouin line, is given by $^{3}$ :

$$
\eta_{v}(\omega)=\eta_{v C}(0)+\frac{\left(c_{P}-c_{V}\right) c_{I}}{\left(c_{V}-c_{I}\right) c_{P} \chi_{S}} \int_{0}^{\infty} d t \exp (-i \omega t) \Phi(t)
$$

where $\eta_{v C}(0)$ is the translational contribution to the bulk viscosity, $c_{V}, c_{\boldsymbol{P}}$, and $c_{\boldsymbol{I}}$ are the specific heats at constant volume and pressure and of the internal modes, $\chi_{s}$ is the adiabatic compressibility and $\Phi(t)$ is the correlation function of the fluctuations of the internal energy. The second term of Eq.6 represents the additional contribution of the internal process to the bulk viscosity and thus to the width of the Brillouin line.

If the $\Phi(t)$ function is exponential:

$$
\Phi(t)=\exp (-t / \tau)
$$

then this additional contribution is given by:

$$
\Delta \eta_{v}(\omega)=\frac{\left(c_{P}-c_{V}\right) c_{I}}{\left(c_{V}-c_{I}\right) c_{P} \chi_{S}} \frac{\tau}{1+i \omega \tau}
$$

\section{Experimental}

\section{Materials}

BMPC and BMMPC synthesized at the Max Planck Institute for Polymer Research, Mainz have the liquid-glass transitions temperatures of 245 and $265 \mathrm{~K}$, respectively ${ }^{15,16,20}$. In order to remove macroscopic impurities (dust) the samples were filtered into dust-free light scattering cells above their melting temperatures of 331 and $346 \mathrm{~K}$, respectively, using Millipore filters 
of pore size of $0.22 \mu \mathrm{m}$. All measurements were performed at temperatures below $350 \mathrm{~K}$ in order to avoid thermal decomposition of the samples. Ortho-terphenyl (OTP) of 97.5\% purity (Merck-Schuchardt) was purified by recrystallization from methanol solutions and vacuum distillation into the dust-free light scattering cells. The purified sample was characterized by the glass transition temperature of $243 \mathrm{~K}$ and a melting temperature of $329 \mathrm{~K}$.

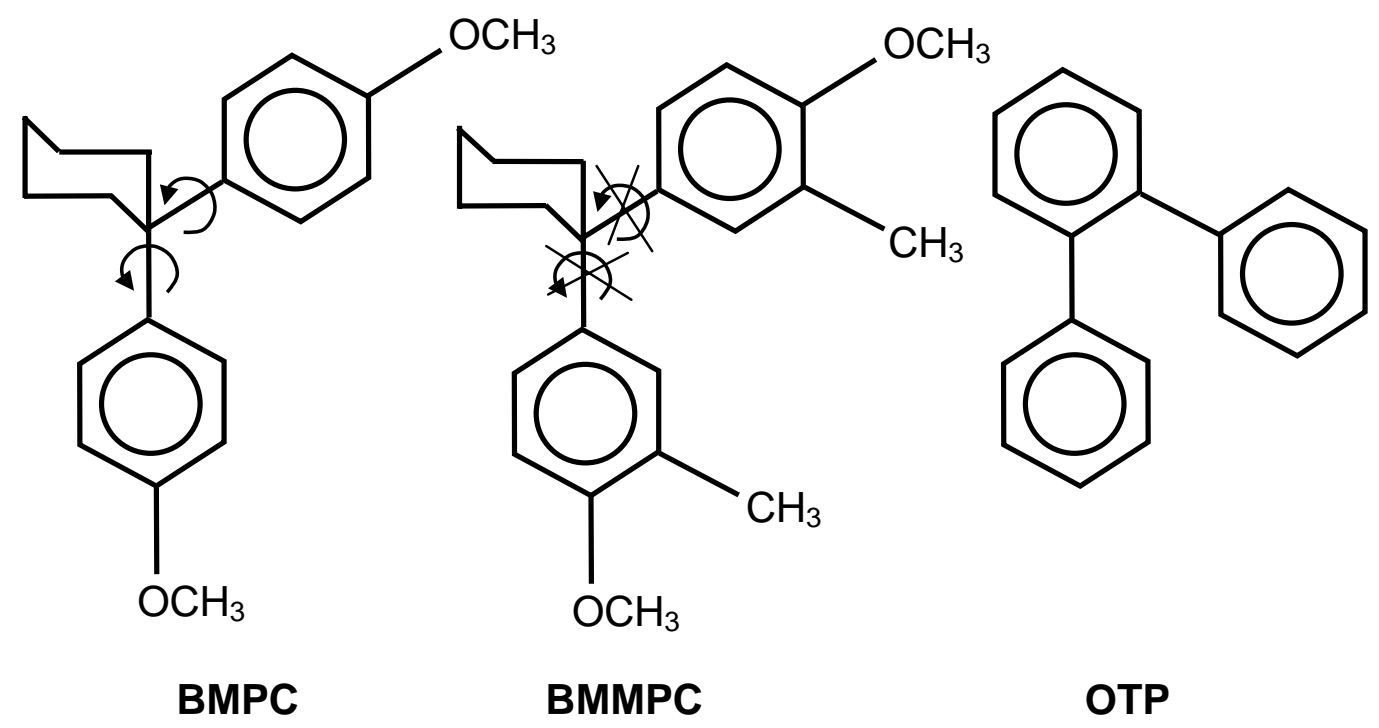

Fig 1: The chemical structures of BMPC (left), BMMPC (middle) with indicated rotations of the phenyl ring, and OTP (right).

\section{Experimantal setup}

Polarized (VV) Rayleigh-Brillouin spectra were measured in the $90^{\circ}$ and back-scattering geometry using a 6-pass Tandem Fabry-Perot interferometer (JRS Instruments, Switzerland). A Nd:YAG laser (Coherent DPSS 532-200) operating at $\lambda=532 \mathrm{~nm}$ at a power of $200 \mathrm{~mW}$ and Ar-ion laser (Spectra Physics, Model 2060, etalon, BeamLock) at $\lambda=514.5 \mathrm{~nm}$ and a power not exceeding $200 \mathrm{~mW}$ were used as incident light sources. Pressure dependent studies were performed in a home-made high pressure cell operating in the pressure range of 1-2000 bar. The experimental high pressure setup was described in more detail elsewhere ${ }^{21}$. 


\section{Data analysis}

Since no theoretical form of the entire Rayleigh - Brillouin spectrum is available for liquids with structural and intramolecular relaxations, we used a simple approach in the analysis of our data, in order to obtain the position $\omega_{\mathbf{B}}$ and the width of $\Gamma_{\boldsymbol{B}}$ of the Brillouin lines. Basically the experimental spectra were fitted using a model function, consisting of the central Rayleigh peak and two symmetric Brillouin peaks according to eq.9. This model function was convoluted with the experimental instrumental function according to eq.2. The instrumental function was determined from a reference beam measurement. For the central peak a sum of a Cole-Davidson function ( $\alpha$-relaxation) and a Lorentzian (fast internal process) was used. The Brillouin peaks were described by Lorentzians (last term in eq.9). The normal Lorenzian form was modified according to Berne and Pecora ${ }^{19}$ in order to account for non-Lorenzian corrections and rendering the Brillouin doublets asymmetric. Thus, the model function has the form:

$$
\begin{aligned}
I(\omega)= & I_{d a r k}+\frac{I_{\alpha}}{2 \pi \omega} \operatorname{Im}\left[1-\left(\frac{1}{1+2 \pi i \omega \tau_{\alpha}}\right)^{\beta_{C D}}\right] \\
& +I_{f} \frac{\Gamma_{f}}{4 \omega^{2}+\Gamma_{f}^{2}}+I_{B} \cdot\left[\frac{\Gamma_{B}-s\left(\omega-\omega_{B}\right)}{4 \cdot\left(\omega-\omega_{B}\right)^{2}+\Gamma_{B}^{2}}+\frac{\Gamma_{B}+s\left(\omega+\omega_{B}\right)}{4 \cdot\left(\omega+\omega_{B}\right)^{2}+\Gamma_{B}^{2}}\right]
\end{aligned}
$$

Here $I_{d a r k}$ denotes the baseline of the measured spectrum, $I_{\alpha}, I_{f}$ and $I_{B}$ are the intensities of the $\alpha$-relaxation, the fast process and Brillouin lines, respectively, $\Gamma_{\mathrm{f}}$ is the width of the fast process, $\omega_{\mathrm{B}}$ and $\Gamma_{\mathrm{B}}$ are the position and width of the Brillouin lines, respectively. Parameter $s$ determines the assymetry of the Brillouin peaks. 


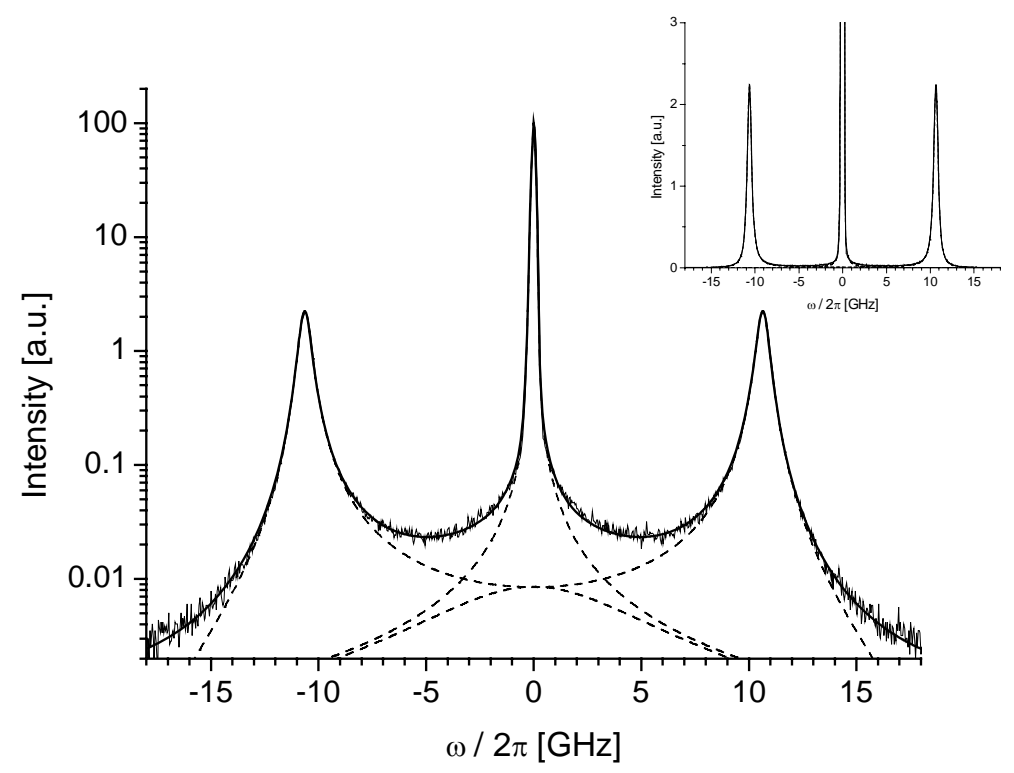

Fig.2: The longitudinal Brillouin spectrum of BMMPC measured at $\theta=90^{\circ}, \lambda=514.5 \mathrm{~nm}$, ambient pressure and $20^{\circ} \mathrm{C}$ temperature. Full line is the fit of eq. 9 to the data. Dashed lines give different contributions to the full expression according to eq.9. The inset shows the same data in linear scale (central line cut) to demonstrate how sharp the Brillouin lines really are.

From a fit of eq. 9 to our experimental data the Brillouin shift $\omega_{\mathrm{B}}$ and Brillouin linewidth $\Gamma_{\mathrm{B}}$ could be obtained. An example of this data analysis is given in Fig.2. Any kind of more sophisticated analysis would likely give access to more parameters, but since this paper is only concerned with interpretations on the level of shift and linewidth we have chosen this rather simple approach. Moreover, the inset in Fig.2 clearly demonstrates that both these parameters are very well defined regardless of the model chosen for the whole spectrum, because the Brillouin lines are very sharp and high compared to the level of their wings. 


\section{Results}

\section{BMPC and BMMPC}

The effects of temperature and pressure on the polarized Brillouin spectra of BMMPC are shown in Figs. 3 and 4, respectively. The central line is the reference beam record used as a measure of the instrumental resolution function. As can be seen, with decreasing temperature or increasing pressure the longitudinal Brillouin peak is moving to higher frequencies and is getting narrower. This is illustrated in Fig.5, where the position of the maximum is plotted for BMPC and BMMPC versus temperature for different pressures from 1 to 1200 bar. In Fig.6 the same data is plotted versus pressure at the same temperatures.

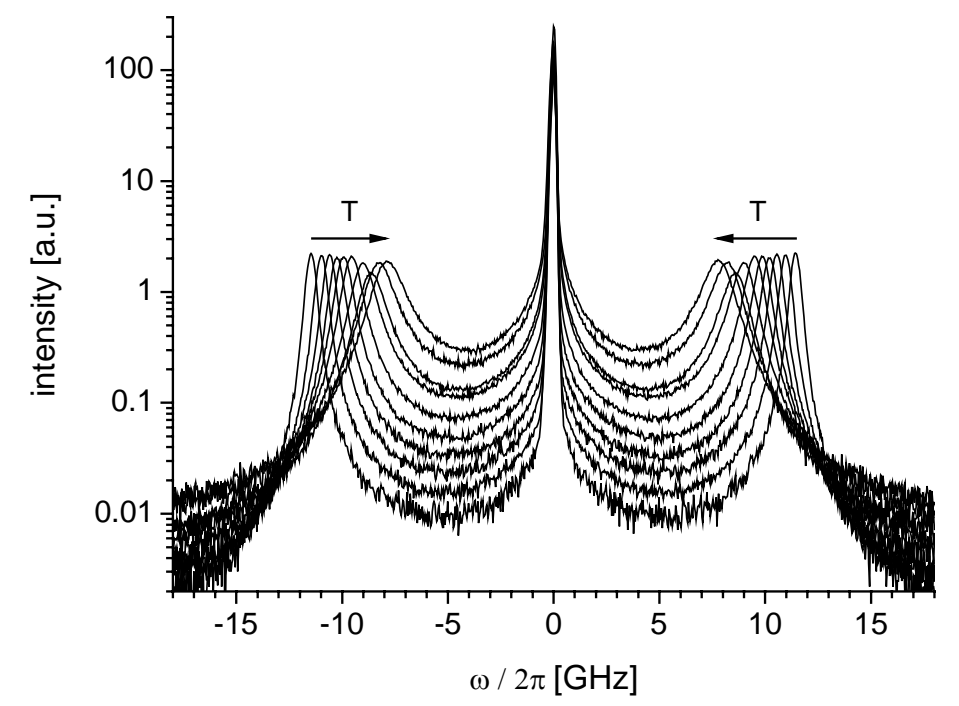

Fig.3: Temperature dependence of the longitudinal Brillouin spectra of BMPC measured at $\theta=90^{\circ}, \lambda=514.5 \mathrm{~nm}$, ambient pressure, at temperatures $\left[{ }^{\circ} \mathrm{C}\right]:-32.1,-18.3,-9.0,0.6$, $10.1,19.8,34.3,44.2,53.9,63.6$, in the order indicated in the figure by two arrows. 


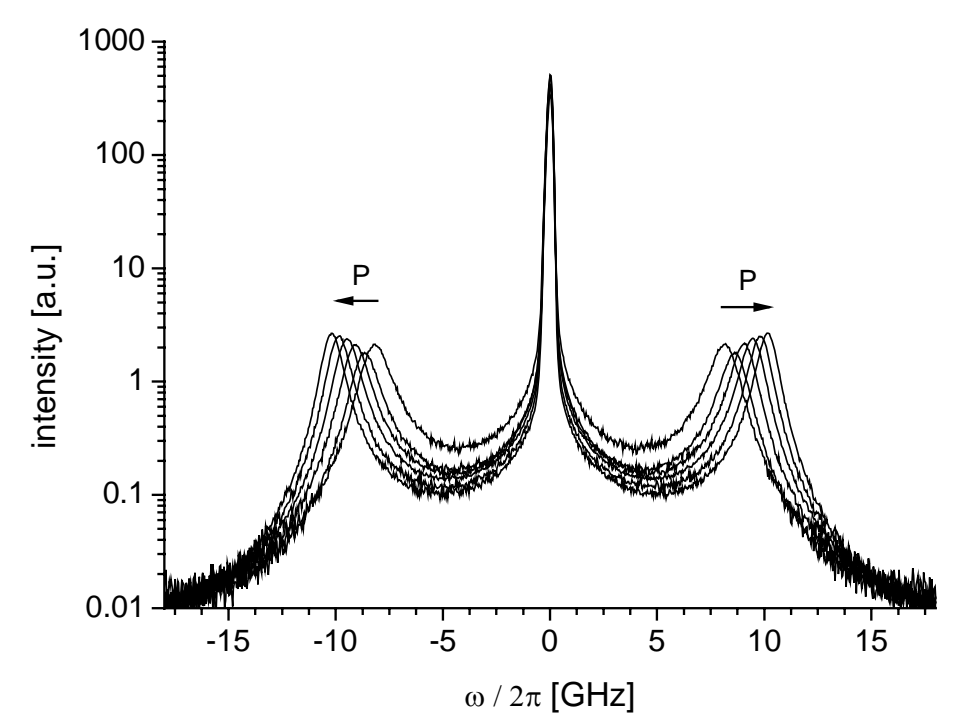

Fig.4: Pressure dependence of the longitudinal Brillouin spectra of BMPC measured at $\theta=$ $90^{\circ}, \lambda=514.5 \mathrm{~nm}$, at a temperature of $20^{\circ} \mathrm{C}$ and pressures of $1,200,400,600,800$ and $1000 \mathrm{bar}$, as indicated in the figure.

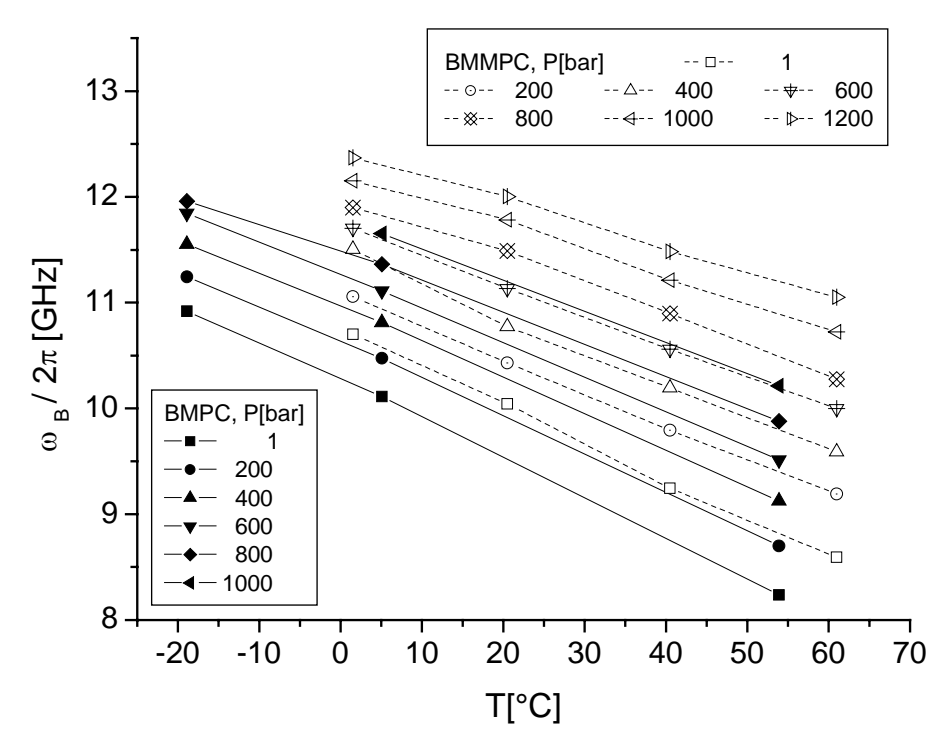

Fig.5: Temperature dependence of the position of the longitudinal Brillouin peak for BMPC and BMMPC obtained from isobaric experiments at $\theta=90^{\circ}, \lambda=514.5 \mathrm{~nm}$ and at pressures in bar as indicated in the legends. The solid symbols and lines correspond to BMPC and open symbols and dashed lines correspond to BMMPC. Lines are guides to the eye. 


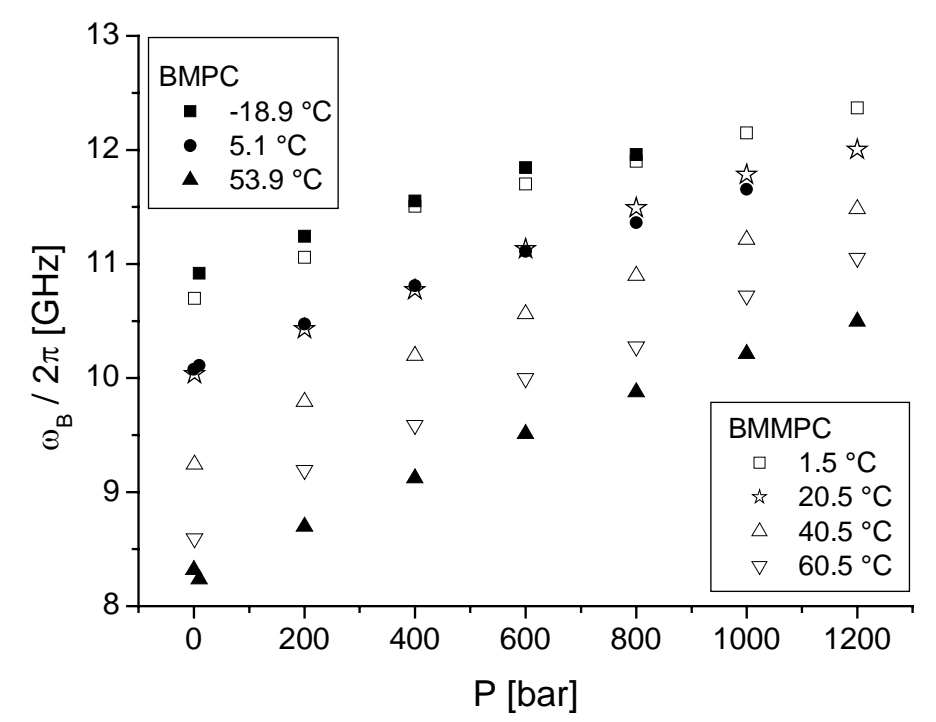

Fig.6: Pressure dependence of the position of the longitudinal Brillouin peak for BMPC and BMMPC obtained from isothermal experiments at $\theta=90^{\circ}, \lambda=514.5 \mathrm{~nm}$ and at temperatures indicated in the figure. The solid symbols correspond to BMPC and open symbols correspond to BMMPC.

A combined temperature and pressure dependence of $\Gamma_{\boldsymbol{B}}$ for BMPC and BMMPC is shown in Fig.7. 


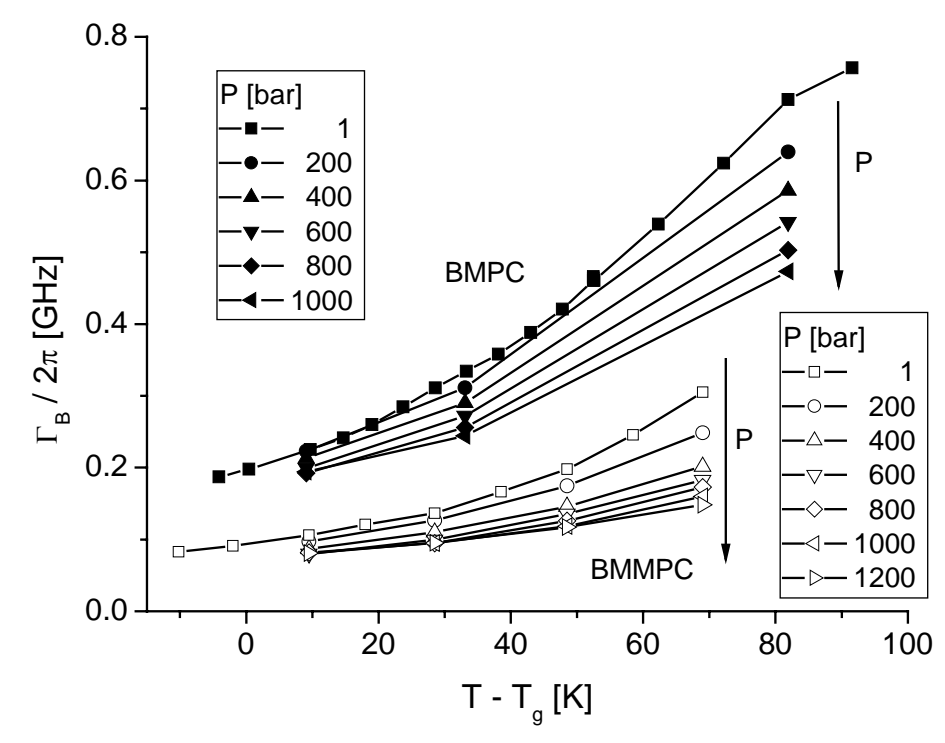

Fig.7: A combined temperature dependence of the width of the longitudinal Brillouin peak $\Gamma_{B}(P)$ for BMPC $\left(T_{g}=245 \mathrm{~K}\right)$ and BMMPC $\left(T_{g}=265 \mathrm{~K}\right)$ measured at $\theta=90^{\circ}, \lambda=514.5 \mathrm{~nm}$ and at pressures indicated in the figure.

As it is evident from Fig.7, the width of the longitudinal Brillouin peak for BMPC is always larger than that for BMMPC, even after correction for the difference between $T_{g}$ 's. Additionally, at the glass transition temperatures, $T_{g}$, the value of $\Gamma_{B} / 2 \pi$ is still substantial and amounts to about $0.2 \mathrm{GHz}$ for $\mathrm{BMPC}$ and is much smaller, $0.09 \mathrm{GHz}$ for BMMPC, respectively.

It has been shown earlier ${ }^{15,16}$ that in BMPC two internal relaxations are present: the phenyl flip and rotation of the $-\mathrm{OCH}_{3}$ group, while in the chemically very similar BMMPC these internal relaxations are no longer possible and are replaced by low amplitude librational motions. Thus, this behavior of $\Gamma_{\boldsymbol{B}}$ for BMPC can be attributed to the presence of a strong internal relaxation - the phenyl flip and the rotation of the $-\mathrm{OCH}_{3}$ group in this molecule. In the case of BMMPC a weaker librational dynamics leads to a smaller broadening of the longitudinal Brillouin line. Obviously, the structural relaxation (density fluctuations) in these supercooled liquids can also contribute to the damping of the longitudinal phonons and 
broadening of the longitudinal Brillouin peaks. In order to separate the contributions of the structural relaxation and internal dynamics to the $\Gamma_{\boldsymbol{B}}$, the pressure dependence of the polarized Brillouin spectra was also measured.

\section{OTP}

It is interesting to compare the results of the polarized Brillouin studies on BMPC and BMMPC with the corresponding data for the well characterized van der Waals glass forming liquid ortho-terphenyl (OTP). Temperature dependence of the position and width of the longitudinal Brillouin lines in OTP was measured in a broad temperature range $\mathrm{e}^{22,23}$. It was, however, impossible to explain the Brillouin width as solely due to the well characterized structural $(\alpha-)$ relaxation. Consequently, OTP is considered to act as a molecule with no phenyl flip motion, similar to the beforehand mentioned BMMPC. This finding is supported by Debus et al. $^{24}$ in a detailed incoherent quasielastic neutron scattering study. A more detailed analysis of the Rayleigh-Brillouin spectra of OTP revealed the existence of additional fast processes of practically temperature independent relaxation times in the picosecond range $e^{8,10,11,14,25-28}$. It was shown ${ }^{14}$, that the fast process can be observed in both supercooled liquid and in crystalline OTP and thus, very likely is of intramolecular character. Taking the fast process into account resulted in much better fits of the Rayleigh-Brillouin spectra ${ }^{10,11}$. No systematic studies of the pressure dependence of the Brillouin shift and width were made for OTP so far.

In our experimental studies we were mainly interested in the temperature and pressure dependence of the Brillouin spectra in the range between the glass transition temperature $T_{\boldsymbol{g}}$ and the melting temperature $T_{m}$, i.e. below the maximum of the acoustic attenuation coefficient. The aim of our study was the determination of the contribution of the intra- and inter-molecular relaxations to the Brillouin line width. 
The corresponding temperature and pressure dependent data measured in the pressure range from 1 to 2000 bar are shown in Figs. 8-10. As one can see in Fig.10, the pressure dependence of $\Gamma_{\boldsymbol{B}}$ in OTP is qualitatively similar to that of BMPC and BMMPC, cf. Fig.7.

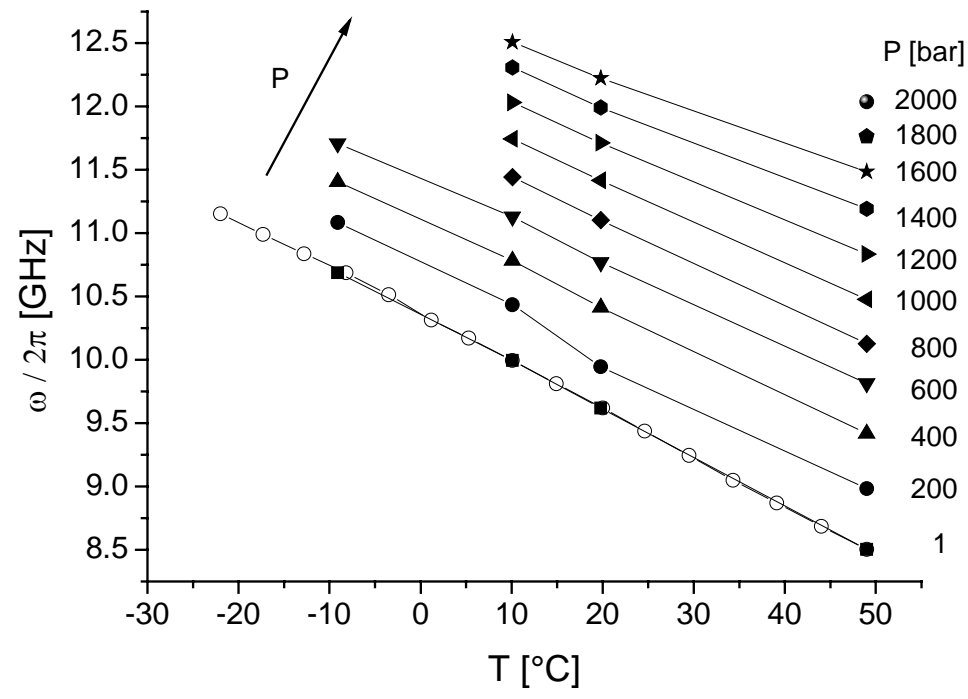

Fig.8: Temperature dependence of the position $\omega_{\mathbf{B}}$ of the polarized Brillouin peak measured for OTP at $\theta=90^{\circ}, \lambda=514.5 \mathrm{~nm}$ at pressures indicated (full symbols). Hollow symbols indicate a more detailed series measured at ambient pressure.

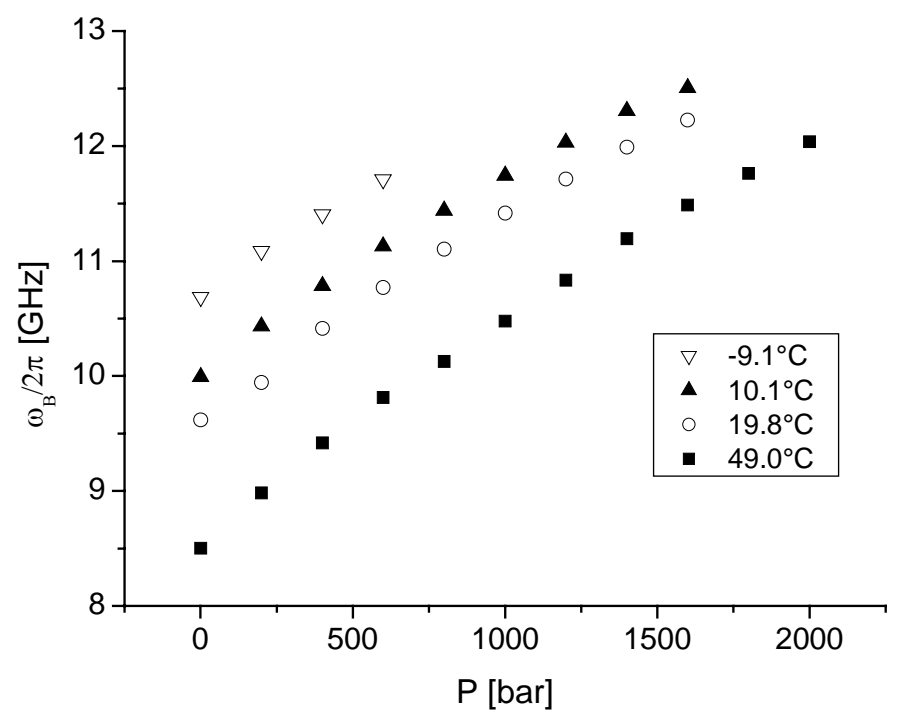

Fig.9: Pressure dependence of the position $\omega_{\boldsymbol{B}}$ of the polarized Brillouin peak measured for OTP at $\theta=90^{\circ}, \lambda=514.5 \mathrm{~nm}$, at temperatures indicated. 


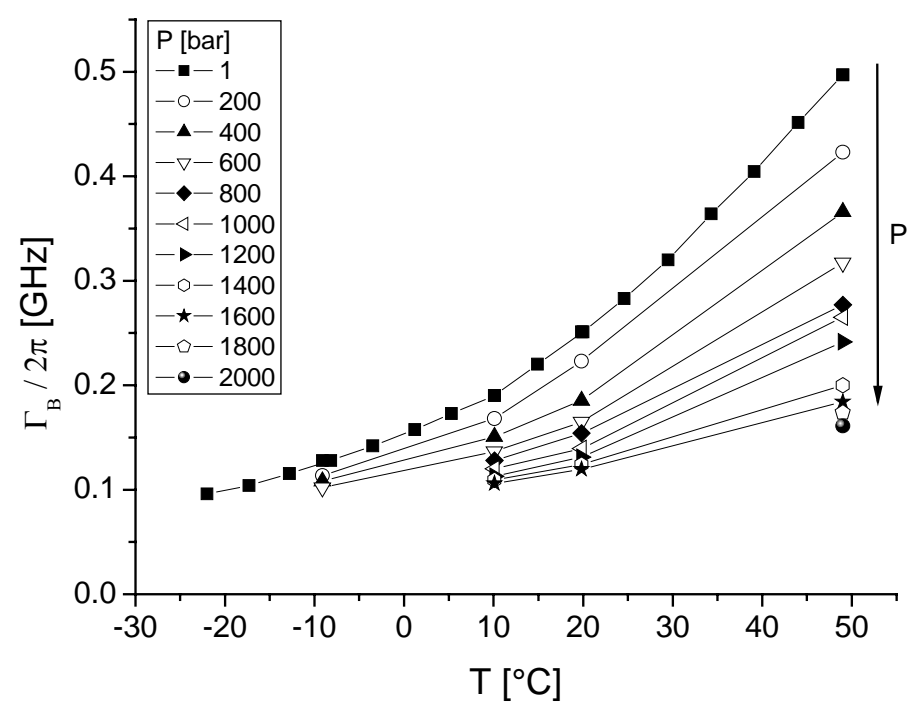

Fig.10: Temperature dependence of the width $\Gamma_{\boldsymbol{B}}$ of the polarized Brillouin peak measured for OTP at $\theta=90^{\circ}, \lambda=514.5 \mathrm{~nm}$, at pressures indicated.

Figures 9 and 10 include also the data for the temperature variation of $\Gamma_{\mathbf{B}}$ and $\omega_{\mathbf{B}}$ for OTP at 1 bar. This temperature dependence of the position $\omega_{\mathbf{B}}$ and width $\Gamma_{\mathbf{B}}$ of the Brillouin peak is in a good agreement with previous studies ${ }^{11,14,22,23}$.

\section{Discussion}

Temperature dependence of the half width at half height of the longitudinal Brillouin peak, $\Gamma_{\mathrm{B}}$, is shown for BMPC, BMMPC and OTP at ambient pressure in Fig.11. One can clearly see that the Brillouin width at the same temperature separation from $T_{\boldsymbol{g}}$ is substantially larger for BMPC, a molecule with strong intramolecular relaxations, than for BMMPC and OTP, where the internal relaxation processes are much weaker. 


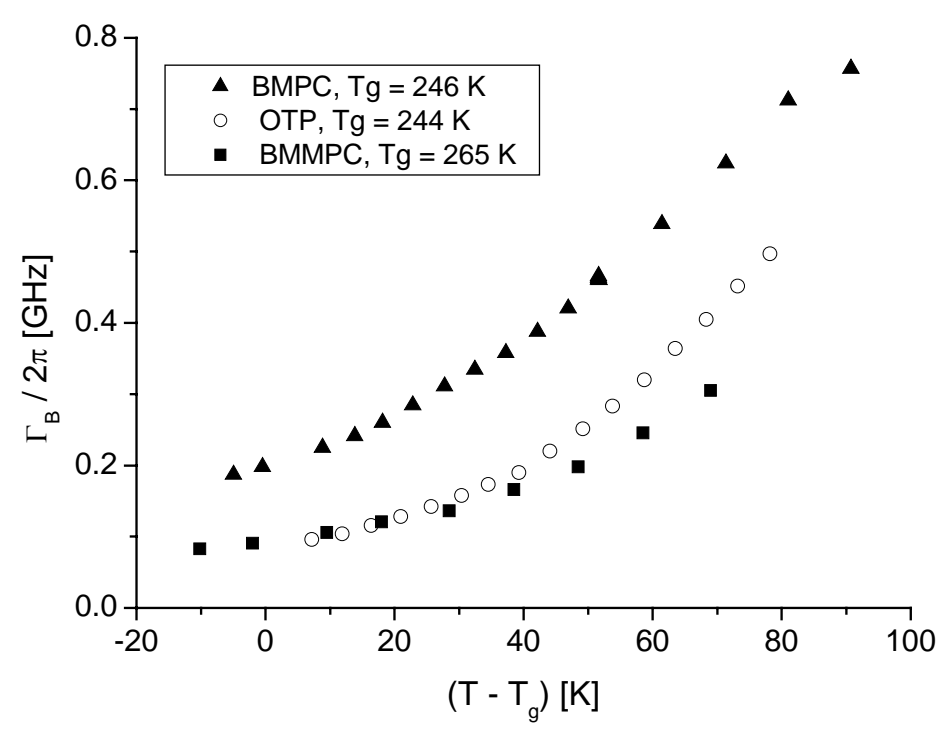

Fig.11: The temperature dependence of the half width at half height of the longitudinal Brillouin peak, $\Gamma_{\boldsymbol{B}}$, for BMPC, BMMPC and OTP measured at $\theta=90^{\circ}, \lambda=514.5 \mathrm{~nm}$ and at ambient pressure.

The pressure dependence of $\Gamma_{\boldsymbol{B}}$ for BMMPC and BMPC at selected temperatures is shown in Fig. 12.

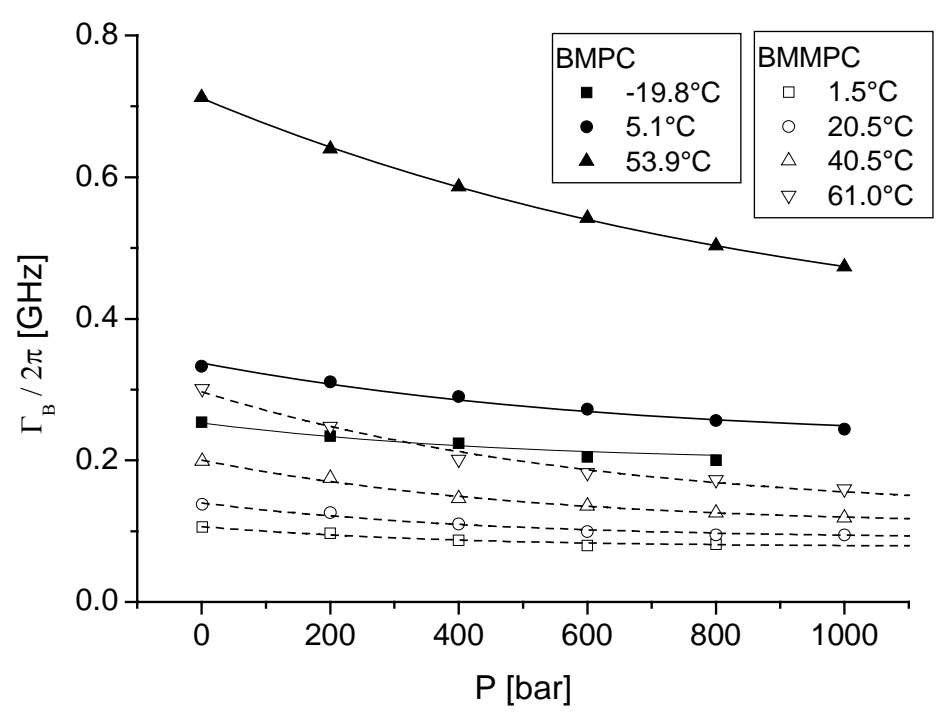

Fig.12. The pressure dependence of $\Gamma_{\boldsymbol{B}}$ for BMPC and BMMPC measured at $\theta=90^{\circ}$, $\lambda=514.5 \mathrm{~nm}$ and at selected temperatures indicated in the figure. 


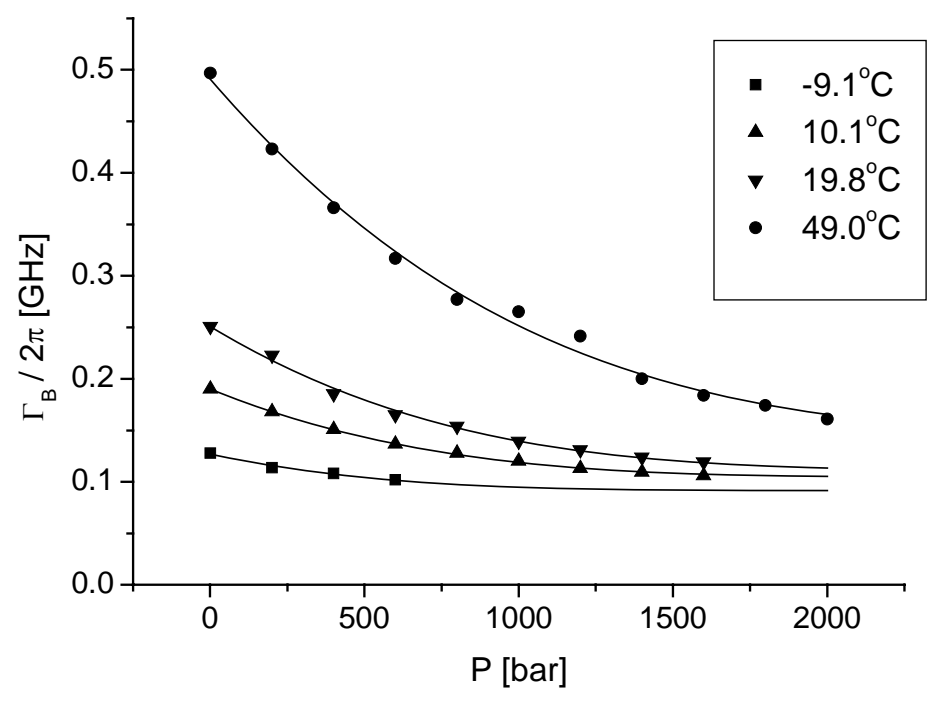

Fig.13. The pressure dependence of $\Gamma_{\boldsymbol{B}}$ for OTP measured at $\theta=90^{\circ}, \lambda=514.5 \mathrm{~nm}$ and at selected temperatures indicated in the figure.

As we can see in Figs.12 and 13, in the high pressure range at all temperatures studied the value of the width of the longitudinal Brillouin peak $\Gamma_{B}(P)$ in BMPC, BMMPC and OTP reaches a plateau, indicating that further increase of pressure is not changing the $\Gamma_{\boldsymbol{B}}$ value any more. The value of $\Gamma_{\boldsymbol{B}}$ at the plateau is increasing with increasing temperature. Additionally, the pressure dependence of $\Gamma_{\boldsymbol{B}}$ is the strongest at the highest temperature and very weak at the lowest temperature close to $T_{\boldsymbol{g}}$. This behavior can be explained in the following way: The high pressure plateau of the $\Gamma_{B}(P)$ corresponds to the contribution of the internal relaxations, since these processes should not depend on pressure (in this pressure range) but only on temperature. The contribution of the structural relaxation to the $\Gamma_{B}(P)$ can be estimated from the difference between the value of $\Gamma_{B}(P)$ at a given pressure and the plateau value. This contribution is the highest at the highest temperature and the lowest pressure studied where the structural relaxation time is the shortest and thus the phonon damping - most efficient. The main difference between BMPC and BMMPC/OTP is that the value of $\Gamma_{\mathbf{B}}$ at the plateau at the lowest temperature close to $T_{\boldsymbol{g}}$ for BMPC is approximately a factor of two higher than 
that for BMMPC and OTP. This difference may be attributed to the presence of strong internal relaxations in BMPC, the phenyl flip and rotation of the $-\mathrm{OCH}_{3}$ group which are replaced by weaker librational processes in the other two molecules.

The temperature dependence of the structural relaxation time is usually expressed in the form of a Vogel-Fulcher-Tammann (VFT) formula:

$$
\tau_{s}(T)=\tau_{\infty} \exp \left(\frac{D_{T} T_{0}}{T-T_{0}}\right)
$$

where $\tau_{\infty}$ is the high temperature limit of $\tau_{\mathrm{s}}, D_{T}$ is the so called strength parameter and $T_{0}$ is called the VFT temperature. In analogy to that, the pressure dependence of $\tau_{\mathrm{s}}$ was expressed in terms of the pressure analog of the VFT formula ${ }^{29}$ :

$$
\tau_{s}(P)=\tau_{0} \exp \left(\frac{D_{P} P}{P_{0}-P}\right)
$$

where $\tau_{0}$ is equal to $\tau_{\mathrm{s}}$ at ambient pressure, $D_{P}$ and $P_{0}$ are analogs of $D_{T}$ and $T_{0}$, respectively. We applied this scheme to characterize the contributions of inter-molecular relaxations to the broadening of the longitudinal Brillouin peaks in a more quantitative way. In order to do that, we fitted the pressure dependent $\Gamma_{B}(P)$ using the formula

$$
\Gamma_{B}(P)=\Gamma_{0}(T)+A(T) \exp \left(\frac{-D_{P} P}{P_{0}-P}\right)
$$

The values of $P_{0}$ corresponding to pressure dependence of respective $\tau_{\mathrm{s}}$ where taken from previous studies on these systems ${ }^{30-32}$. From this analysis, temperature dependent values of the contributions of the intra- $\left(\Gamma_{0}(T)\right)$ and structural (inter-molecular) $(A(T))$ relaxations were obtained and are shown in Figs.14 and 15. 


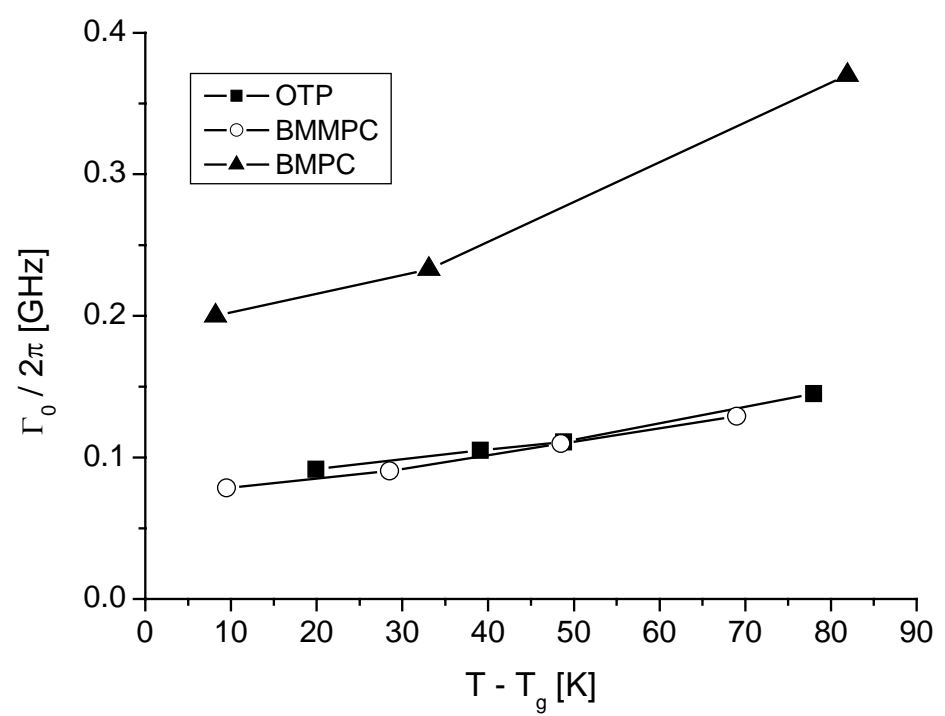

Fig.14: Temperature dependence of the estimated contribution of the intramolecular relaxations to the width of the longitudinal Brillouin peak in BMPC, BMMPC and OTP.

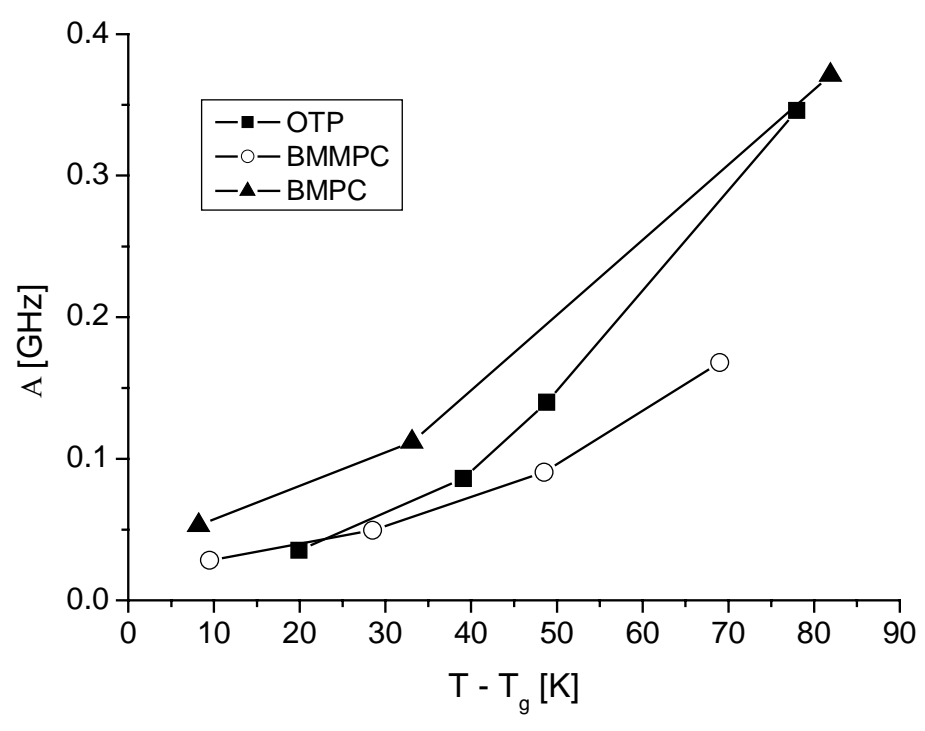

Fig.15: Temperature dependence of the estimated contribution of the structural $(\alpha-)$ relaxation to the width of the longitudinal Brillouin peak in BMPC, BMMPC and OTP.

An additional indication of fast relaxation processes present in supercooled BMMPC and OTP can be found in the positron annihilation studies ${ }^{33,34}$. In these liquids a weak increase of free volume with increasing temperature was observed below $T_{g}$. This effect can be explained 
by coupling of intra-molecular relaxations to the center of mass motion of the molecules (structural relaxation). These fast relaxation processes can also be observed in the crystalline samples $^{33,14}$.

Temperature and pressure dependence of the relaxation times of all processes in comparison to the Brillouin shift are summarized for BMPC in Fig.16.

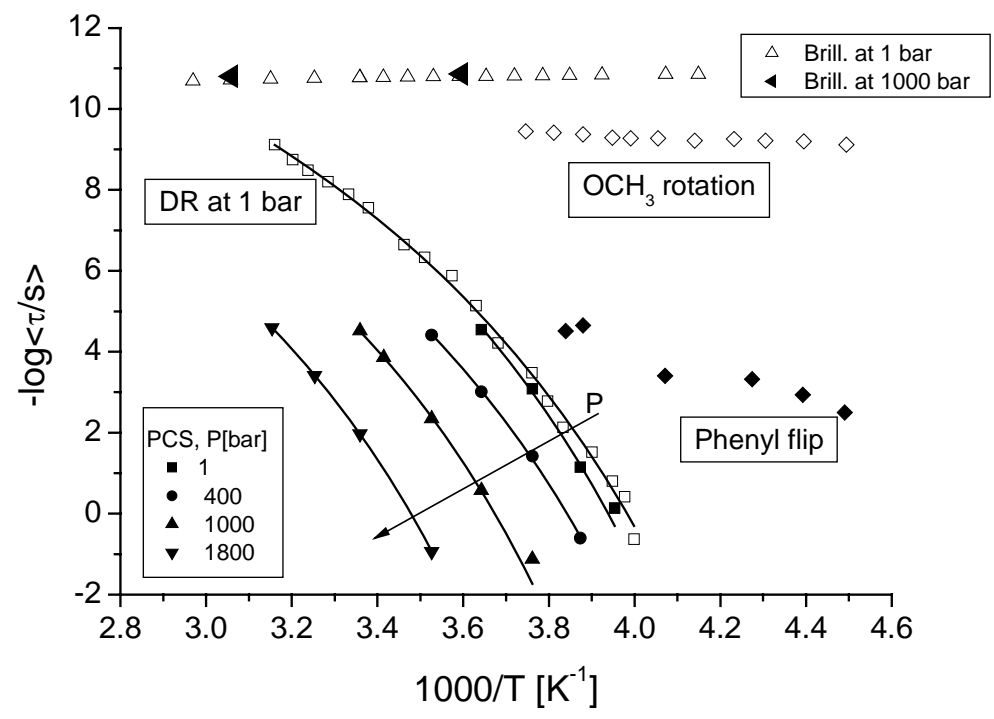

Fig.16: Temperature and pressure dependence of the relaxation times of the structural $(\alpha-)$ relaxation $^{15,35}$ and the Brillouin shift as well as temperature dependence of the relaxation times of the intra-molecular processes ${ }^{15}$.

While the structural relaxation times strongly depend on pressure (and temperature), the pressure dependence of the Brillouin peak frequency and probably the relaxation times of the intra-molecular relaxations is very weak and their temperature dependence is Arrhenius-like with a low activation energy. It is clearly seen that at all temperatures and pressures studied the relaxation times of the intra-molecular processes (the phenyl flip and the $-\mathrm{OCH}_{3}$ rotation) are much closer to the inverse Brillouin frequency than that of the structural $(\alpha-)$ relaxation. The contribution of a given relaxation process to the damping of phonons at the Brillouin frequency depends also on the distribution of its relaxation times. In order to illustrate these 
contributions of all three processes in BMPC we have plotted their susceptibilities $\chi ”(\omega) \approx \omega S(\omega)$ for two temperatures in Fig. 17.

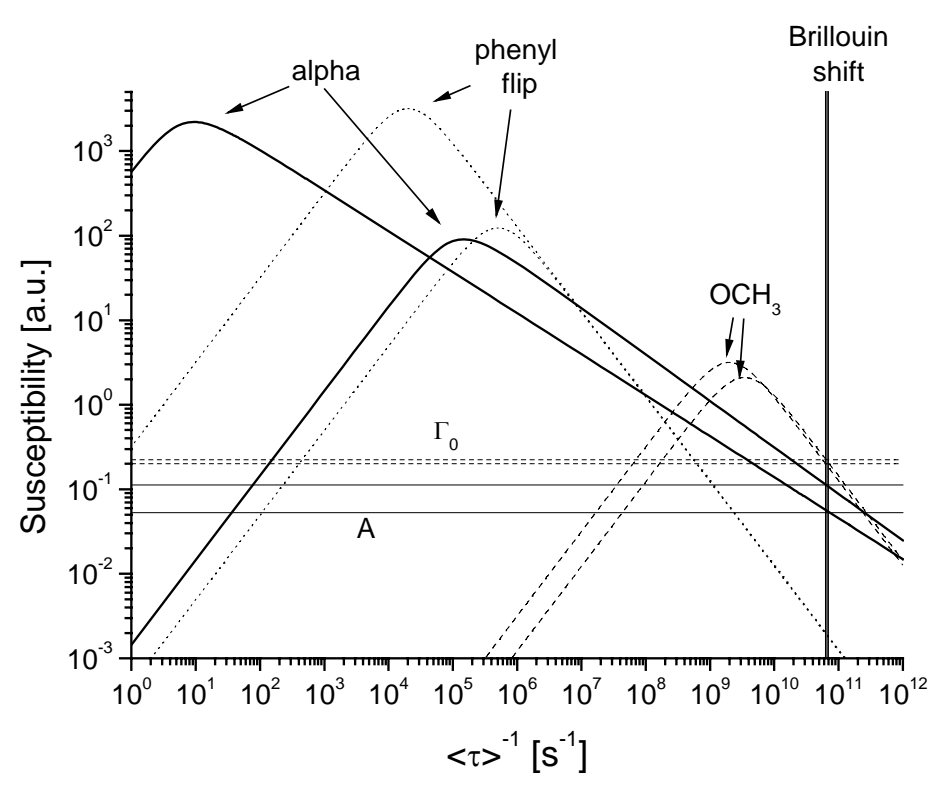

Fig.17: Susceptibilities of the structural $(\alpha-)$ relaxation - solid lines, and internal processes: the phenyl flip - dotted lines and the $-\mathrm{OCH}_{3}$ group rotation - dashed lines for BMPC at temperatures of -18.9 and $5.1^{\circ} \mathrm{C}$.

In this figure the structural $(\alpha-)$ relaxation is taken in the usual form of the Cole-Davidson process, while the phenyl flip and the $-\mathrm{OCH}_{3}$ group rotation are represented by Lorentzians, according to the literature data ${ }^{15}$. The positions of the peaks in the susceptibility spectra corresponds to the values of the processes given in the activation plot (Fig.16). The amplitudes of the $\alpha$ - and $-\mathrm{OCH}_{3}$ processes were adjusted such that their susceptibilities at experimental Brillouin frequencies were proportional to their contributions to the phonon damping, $A(T)$ and $\Gamma_{0}(T)$ (Eq.12), respectively. The amplitudes of the phenyl flip process were set equal to those of the corresponding (same temperature) $\alpha$-process, which we regarded as the upper reasonable limit. 
It can be clearly seen that the contributions of different processes to the broadening of the Brillouin peak depend on their distance from the Brillouin frequency $\omega_{\mathrm{B}}$, their amplitude at the peak and the shape of the spectrum (Cole-Davidson, Lorentzian, etc.). The contribution of the structural relaxation increases with increasing temperature due to the substantial increase of its relaxation frequency. Although the relaxation rate of the phenyl flip is much closer to $\omega_{\mathrm{B}}$, than that of the $\alpha$-process, it does not contribute because of its Lorentzian shape. The strongest contributions at both temperatures are due to the $-\mathrm{OCH}_{3}$ group rotation despite of the Lorentzian form of the relaxation spectrum, because the relaxation rate of this process is the closest one to $\omega_{\mathrm{B}}$.

Thus, the expected contribution of the internal processes to the damping of the longitudinal phonons and the broadening of the isotropic Brillouin peak will be substantial or even dominant at all temperatures and pressures. This is corroborated by our results, Figs. 14,15, showing the estimated contributions of the intra- and inter-molecular relaxations to the damping (broadening) of longitudinal phonons (Brillouin peaks). Thus any detailed analysis of the width and shape of the Brillouin peaks for materials with internal (fast) relaxations must explicitly take the contributions of these processes into account.

\section{Conclusions}

Propagation and damping of longitudinal phonons were studied in two chemically very similar van der Waals glass forming liquids: BMPC and BMMPC by means of Brillouin spectroscopy in a broad temperature and pressure range. In BMPC, in addition to the usual structural $(\alpha-)$ relaxation, strong intra-molecular relaxations are present, while in BMMPC these internal processes are very strongly hindered by the chemical modification. The results were compared with data obtained for another similar liquid, OTP. 
From the analysis of the isotropic Brillouin spectra we found that the internal relaxations contribute substantially to the damping (broadening) of longitudinal phonons (isotropic Brillouin peaks).

We have shown, that the contribution of intra- and inter-molecular processes to the width of the Brillouin peaks can be estimated from pressure dependent Brillouin studies because the structural relaxation times (and its contribution to the damping of phonons) depend very strongly on pressure, while the intra-molecular processes are practically pressure independent in the pressure range used.

Since the relaxation times of intra-molecular (fast) processes are usually much closer to $1 / \omega_{\mathrm{B}}$, than that of the structural relaxation, the internal processes strongly contribute to the damping of longitudinal phonons and to the width of the isotropic Brillouin peaks and must be explicitly taken into account in the theoretical description of the isotropic Brillouin spectra.

\section{Acknowledgements}

This work was supported by the Polish Ministry of Science and Higher Education (grant No 1 P03B 083 26) and European Network of Excellence "SoftComp" (No. S080118). 


\section{References}

1. A. Aouadi, C. Dreyfus, M. Massor, R. M. Pick, T. Berger, W. Steffen, A. Patkowski, and C. Alba-Simionesco, J. Chem. Phys. 112, 9860 (2000).

2. C. Dreyfus, A. Aouadi, R. M. Pick, W. Steffen, T. Berger, and A. Patkowski, Europhys. Lett., 42, 55 (1998).

3. R. Zwanzig, J. Chem. Phys., 43, 714 (1965).

4. S. Saito, H. Sasabe, T. Nakajima and K. Yada; J. Polym. Sci. Part A2, 6, 1297 (1968)

5. G. Williams, Trans. Faraday Soc., 62, 2091 (1966)

6. G. Fytas, G. Meier, A. Patkowski and Th. Dorfmüller, Coll. Polym. Sci, 260, 949 (1982)

7. M. Paluch, C. M. Roland, S. Pawlus, J. Zioło and K. L. Ngai, Phys. Rev. Lett, 91, $115701(2003)$

8. W. Steffen, A. Patkowski, G. Meier and E. W. Fischer, J. Chem. Phys., 96, 4171 (1992)

9. A. Patkowski, W. Steffen, G. Meier and E. W. Fischer, J. Non-Cryst. Solids, 172-174, $52(1994)$

10. G. Monaco, D. Fioretto, C. Masciovecchio, G. Ruocco and F. Sette, Phys. Rev. Lett., 82, $1776(1999)$

11. G. Monaco, D. Fioretto, L. Comez, and G. Ruocco, Phys. Rev. E, 63, 061502 (2001)

12. C. H. Wang, X. R. Zhu and J. C. Shen., Mol. Phys., 62, 749 (1987)

13. S. Mossa, G. Monaco and G. Ruocco, Europhys. Lett., 60, 92(2002)

14. G. Monaco, S. Caponi, R. DiLeonardo, D. Fioretto and G. Ruocco, Phys. Rev. E, 62, R7595 (2000)

15. G. Meier, B. Gerharz, D. Boese, and E.W. Fischer, J. Chem. Phys. 94, 3050 (1991)

16. S. Kahle, J. Gapinski, G. Hinze, A. Patkowski and G. Meier, J. Chem. Phys., 122, $074506(2005)$ 
17. A. Patkowski, J. Gapinski, G. Meier, H. Kriegs, A. Le Grand and C. Dreyfus, Europhys. Lett., 73, 607 (2006)

18. R. Zwanzig, in Lectures in Theoretical Physics, edited by W. Brittin (WileyInterscience, New York, 1961), Vol. 3, pp. 106-141; H. Mori, Prog. Theor. Phys., 33, 423(1965)

19. B. J. Berne and R. Pecora, Dynamic Light Scattering (Dover Publications, Inc., Mineola, New York) 2000, p. 243

20. B. Gerharz, G. Meier, and E.W. Fischer, J. Chem. Phys. 92, 7110 (1990)

21. G. Fytas, G. Meier and Th. Dorfmüller, Macromolecules, 18, 993 (1985).

22. Y. Higashigaki and C. H. Wang, J. Chem. Phys., 743175 (1981)

23. C. H. Wang, X. R. Zhu and J. C. Shen, Mol. Phys., 62, 749 (1987)

24. O.Debus, H. Zimmermann, E. Bartsch, F. Fujara, M. Kiebel, W. Petry and H. Sillescu, Chem. Phys. Lett., 180, 271 (1991)

25. E. W. Fischer, G. Meier, T. Rabenau, A. Patkowski, W. Steffen and W. Thönnes, J.NonCryst. Solids, 131-133, 134 (1991)

26. W. Steffen, A. Patkowski, G. Meier and E. W. Fischer, J. Chem. Phys., 96, 4171 (1992)

27. W. Steffen, B. Zimmer, A. Patkowski, G. Meier and E. W. Fischer, J. Non-Cryst. Solids, 172-174, 37(1994)

28. A. Patkowski, M. Matos Lopes and E. W. Fischer, J. Chem. Phys., 119, 1579 (2003)

29. M. Paluch, A. Patkowski, and E.W. Fischer, Phys. Rev. Lett., 85, 2140 (2000).

30. J. Gapinski, M. Paluch, A. Patkowski, Phys. Rev. E., 66, 011501 (2002).

31. A. Patkowski, J. Gapinski, G. Meier, Colloid Polym. Sci., 282, 874 (2004).

32. G. Fytas, T.H. Dorfmüller, C.H. Wang, J. Chem. Phys. 87, 5041 (1983).

33. Sz. Vass, A. Patkowski, E. W. Fischer, K. Süvegh and A. Vertes, Europhys. Lett. 46, $815(1999)$ 
34. B. B. Malhotra and R. A. Pethrick, J. Chem. Soc. Faraday. Trans II, 78, 297 (1982)

35. A. Patkowski, J. Gapinski, and G. Meier, Coll. Polym. Sci., 282, 874 (2004) 